\title{
Torus Models of the Outer Disc of the Milky Way using LAMOST Survey Data
}

\author{
Qiao Wang ${ }^{1 \star}$, Yougang Wang ${ }^{1}$, Chao Liu ${ }^{2}$, Shude Mao ${ }^{3,1,4}$, R. J. Long ${ }^{5,4}$ \\ ${ }^{1}$ Key Laboratory of Computational Astrophysics, National Astronomical Observatories, Chinese Academy of Sciences, Beijing, 100012 China \\ ${ }^{2}$ Key Laboratory of Optical Astronomy, National Astronomical Observatories, Chinese Academy of Sciences, Beijing, 100012 China \\ ${ }^{3}$ Department of Physics, and Center for Astrophysics, Tsinghua University, 100086 Beijing, China \\ ${ }^{4}$ Jodrell Bank Centre for Astrophysics, School of Physics and Astronomy, The University of Manchester, Oxford Road, Manchester M13 9PL, UK \\ ${ }^{5}$ National Astronomical Observatories, Chinese Academy of Sciences, Beijing, 100012 China
}

Accepted XXX. Received YYY; in original form ZZZ

\begin{abstract}
With a sample of 48,161 K giant stars selected from the LAMOST DR 2 catalogue, we construct torus models in a large volume extending, for the first time, from the solar vicinity to a Galactocentric distance of $\sim 20 \mathrm{kpc}$, reaching the outskirts of the Galactic disc. We show that the kinematics of the $\mathrm{K}$ giant stars match conventional models, e.g. as created by Binney in 2012, in the Solar vicinity. However such two-disc models fail if they are extended to the outer regions, even if an additional disc component is utilised. If we loosen constraints in the Sun's vicinity, we find that an effective thick disc model could explain the anti-centre of the MW. The LAMOST data imply that the sizes of the Galactic discs are much larger, and that the outer disc is much thicker, than previously thought, or alternatively that the outer structure is not a conventional disc at all. However, the velocity dispersion $\sigma_{0 z}$ of the kinematically thick disc in the best-fitting model is about $80 \mathrm{~km} \mathrm{~s}^{-1}$ and has a scale parameter $R_{\sigma}$ for an exponential distribution function of $\sim 19 \mathrm{kpc}$. Such a height $\sigma_{0 z}$ is strongly rejected by current measurements in the solar neighbourhood, and thus a model beyond quasi-thermal, two or three thin or thick discs is required.
\end{abstract}

Key words: Galaxy: structure - Galaxy: kinematics and dynamics - Galaxy: disc galaxies: kinematics and dynamics

\section{INTRODUCTION}

It is of great importance to construct dynamical models for the Milky Way (MW) to help us understand the structure and formation of the MW and by implication other spiral galaxies. Compared with such galaxies, the MW has the most extensive observational data. Many stellar kinematic surveys with different magnitude limitations and different observing directions have been carried out, such as the Bulge Radial Velocity Assay (BRAVA, Rich et al. 2007; Kunder et al. 2012), the Abundances and Radial velocity Galactic Origins Survey (ARGOS, Freeman et al. 2013), the Apache Point Observatory Galactic Evolution Experiment (APOGEE, Allende Prieto et al. 2008; Majewski et al. 2013), SDSS (York et al. 2000), the GenevaCopenhagen Survey (GCS, Nordström et al. 2004), RAdial Velocity Experiment (RAVE, Steinmetz et al. 2006), the LAMOST Experiment for Galactic Understanding and Ex-

* E-mail:qwang@nao.cas.cn ploration (LEGUE, Deng et al. 2012), the Global Exploration Strategy (GES, Gilmore et al. 2012) and the GALactic Archeology with Hermes survey (GALAH, De Silva et al. 2015) etc.

Several methods have been applied to construct dynamical models of the MW. Firstly, Jeans modelling is based on velocity moments of the Jeans equation and connects density, potential and kinematics (Jeans 1915, 1919). For example, Xue et al. (2008, 2015), Kafle et al. (2014) and Huang et al. (2016) employed Jeans models to estimate the mass of the MW using SDSS data. Secondly, Schwarzschild's orbit-superposition technique (Schwarzschild 1979) relies on replacing the distribution function by a combination of orbit weights and representative orbits. Wang et al. (2012, 2013) have constructed Schwarzschild models of the Galactic bar using BRAVA data. Thirdly, the Made-to-Measure (M2M) method which is particle based (Syer \& Tremaine 1996) finds the best fit to observations by modifying its particle weights to match the input density and kinematic ob- 
servables. M2M models of the MW have been constructed by Long et al. (2013) (bar) and Portail et al. (2015) (bulge).

More recently, there has been an increasing interest in torus and action-based distribution function methods for modelling the Milky Way (Sanders \& Binney 2016). In these methods, stellar distribution functions are constructed from Hamiltonian mechanics action variables (see Binney \& Tremaine 2008). One of the action methods is referred to as torus modelling (McMillan \& Binney 2008). It replaces the orbits in Schwarzschild method by tori and constructs torus libraries. There are other methods. For example, Binney (2012) connected data from the GenevaCopenhagen survey of the solar neighbourhood to distribution functions by the Stäckel fudge method (Bovy 2015; Sanders \& Binney 2015), and showed in Binney et al. (2014) that their results are consistent with those from the RAVE survey. Their findings also suggest that data extending significantly beyond the solar radius (as our LAMOST data do) are needed in order to better constrain their models.

Piffl et al. (2014) and Binney \& Piffl (2015) extended earlier modelling and developed the DF of the halo, constraining its parameters by using the RAVE survey and SDSS data within $\sim 2.5 \mathrm{kpc}$ around the Sun. The LAMOST survey has observed a few millions of stars along the Galactic anti-centre direction. This allows us to study the outer disc with many more stars than in previous surveys. In particular, given a limiting magnitude of $r=17.8$ mag, LAMOST K giant stars can reach as far as $90 \mathrm{kpc}$ from the Galactic centre (Liu et al. 2014). The LAMOST sample is thus the best data currently available for the study of the stellar distribution function of the Galactic disc which may extend to about $20 \mathrm{kpc}$ from the Galactic centre.

In this paper, we will construct torus models of the MW using LAMOST data. Our aims are two-fold :

(i) to corroborate or otherwise with LAMOST data results from earlier torus models;

(ii) to examine whether and how LAMOST data can be used to constrain better the structure of the MW disc.

The paper is structured as follows. Section 2 describes the LAMOST data. In section 3, we discuss torus modelling, and the algorithms and parameters we use. Results are shown in section 4 and are discussed in section 5 .

\section{DATA SAMPLE}

The Large Area Multi-object Spectroscopic Telescope (LAMOST; also called the Guoshoujing telescope) is a 4metre reflective Schmidt telescope with a 5-degree field-ofview. 4000 fibres are configured on the wide focal plane, allowing one to observe a few thousands objects simultaneously (Cui et al. 2012; Zhao et al. 2012). The LAMOST MW survey targets a few millions of stars with a limiting magnitude of $r=17.8$ mag. It covers most of the sky from dec $=-10^{\circ}$ to $60^{\circ}$ (Deng et al. 2012). Due to the position of the site, during winter, it can efficiently observe the Galactic anti-centre (Yao et al. 2012). Liu et al. (2014) successfully identified $280,000 \mathrm{~K}$ giant stars in the DR1 data. This was later extended to about 450,000 in the DR2 data (Ho et al. 2016). Meanwhile, Carlin et al. (2015) improved
Table 1. The sky area divided into blocks. There are 26 blocks in total. The first 16 blocks cover the solar neighbourhood. Blocks $17-24$ cover $20^{\circ} \times 20^{\circ}$ at the anti-centre of the MW out to $12 \mathrm{kpc}$. The number of $\mathrm{K}$ giants is given in the last column of the table.

\begin{tabular}{|c|c|c|c|c|}
\hline & $\begin{array}{l}\text { longitude range } \\
\text { (deg) }\end{array}$ & $\begin{array}{c}\text { latitude range } \\
\text { (deg) }\end{array}$ & $\begin{array}{l}\text { distance } \\
(\mathrm{kpc})\end{array}$ & counts \\
\hline 01 & $(160,170)$ & $(-20,-10)$ & {$[0,2]$} & 2340 \\
\hline 02 & $(160,170)$ & $(-10,0)$ & {$[0,2]$} & 3922 \\
\hline 03 & $(160,170)$ & $(0,10)$ & {$[0,2]$} & 2778 \\
\hline 04 & $(160,170)$ & $(10,20)$ & {$[0,2]$} & 838 \\
\hline 05 & $(170,180)$ & $(-20,-10)$ & {$[0,2]$} & 1749 \\
\hline 06 & $(170,180)$ & $(-10,0)$ & {$[0,2]$} & 3434 \\
\hline 07 & $(170,180)$ & $(0,10)$ & {$[0,2]$} & 4730 \\
\hline 08 & $(170,180)$ & $(10,20)$ & {$[0,2]$} & 1293 \\
\hline 09 & $(180,190)$ & $(-20,-10)$ & {$[0,2]$} & 385 \\
\hline 10 & $(180,190)$ & $(-10,0)$ & {$[0,2]$} & 3251 \\
\hline 11 & $(180,190)$ & $(0,10)$ & {$[0,2]$} & 4630 \\
\hline 12 & $(180,190)$ & $(10,20)$ & {$[0,2]$} & 1811 \\
\hline 13 & $(190,200)$ & $(-20,-10)$ & {$[0,2]$} & 860 \\
\hline 14 & $(190,200)$ & $(-10,0)$ & {$[0,2]$} & 2323 \\
\hline 15 & $(190,200)$ & $(0,10)$ & {$[0,2]$} & 2732 \\
\hline 16 & $(190,200)$ & $(10,20)$ & {$[0,2]$} & 1667 \\
\hline 17 & $(170,180)$ & $(-10,0)$ & {$[2,3]$} & 831 \\
\hline 18 & $(170,180)$ & $(0,10)$ & {$[2,3]$} & 1212 \\
\hline 19 & $(180,190)$ & $(-10,0)$ & {$[2,3]$} & 446 \\
\hline 20 & $(180,190)$ & $(0,10)$ & {$[2,3]$} & 1200 \\
\hline 21 & $(170,190)$ & $(-10,10)$ & {$[3,4]$} & 2114 \\
\hline 22 & $(170,190)$ & $(-10,10)$ & {$[4,5]$} & 1272 \\
\hline 23 & $(170,190)$ & $(-10,10)$ & {$[5,7]$} & 1184 \\
\hline 24 & $(170,190)$ & $(-10,10)$ & {$[7,12]$} & 669 \\
\hline 25 & $(0,360)$ & $(80,90)$ & {$[0,1.5]$} & 270 \\
\hline 26 & $(0,360)$ & $(80,90)$ & {$[1.5,3]$} & 430 \\
\hline
\end{tabular}

the distance estimation for all stars with stellar parameters, including $\mathrm{K}$ giant stars, to an accuracy of about $20 \%$ (see the appendix). $\mathrm{K}$ giants were chosen as they are bright and detectable at distances far from the Sun. In addition, K giants are present in multiple stellar populations and contain information on different ages and chemistries.

The selection effect in the LAMOST survey is rather simple (Liu et al. 2017a). The targeting selection strategy mostly depends on the apparent magnitude rather than the color index. Therefore, it would not introduce any bias in kinematics. Moreover, although the initial target selection may be slightly altered by the observations and data reduction, only the very blue or red objects, which may have extremely low signal-to-noise ratio at one end of the spectra (blue end for red objects and vice versa), are possibly dropped by these processes. For the $\mathrm{K}$ giant stars used in this work, because their signal-to-noise ratios are quite balanced throughout the whole wavelength coverage, they should not be significantly affected by any systematic bias during observations and data processing. Therefore, the sample should not have substantial systematic bias in the color index. Consequently, there should be no systematic bias in ages or metallicities, since they are mainly associated with color index.

The kinematics of the stellar disc towards the anticentre are studied in this work. Fig. 1 shows the footprint of 


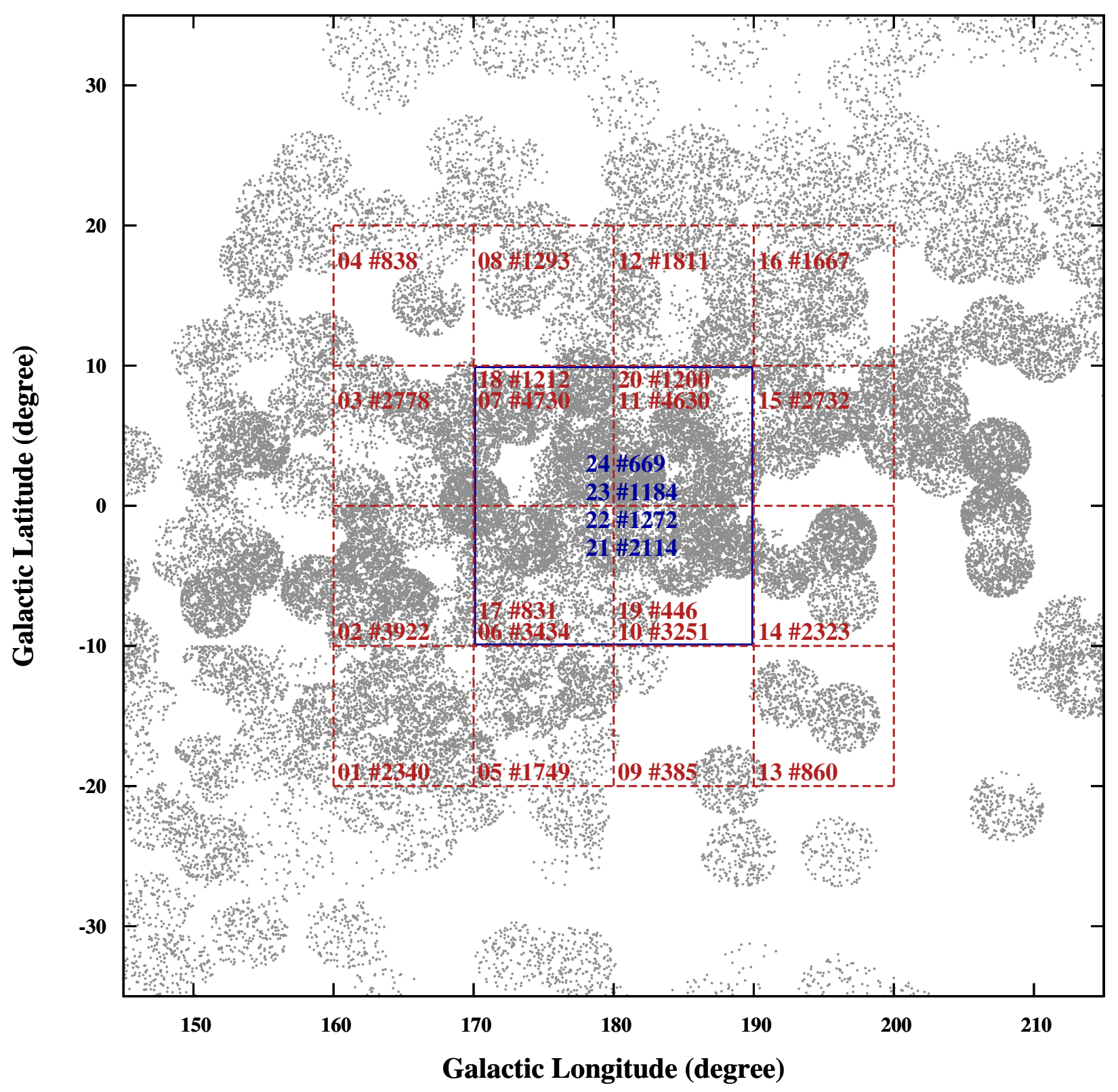

Figure 1. The footprint of the LAMOST survey showing the sky blocks in this work (see Table 1). The labels denote the identifier of the block and the number of stars in the block (after the \#). Blocks $01-16$ occupy the nearest $2 \mathrm{kpc}$ subdivision from the Sun, and each block covers $10 \times 10 \mathrm{deg}^{2}$ sky area. Blocks 17, 18, 19, and 20 cover the same directions as 06, 07, 10, and 11 respectively, but cover the depth of $2 \sim 3 \mathrm{kpc}$. Blocks 21, 22,23 and 24 cover $20 \times 20 \mathrm{deg}^{2}$ of sky area of the Galactic anti-centre from $3 \sim 12 \mathrm{kpc}$ and the blue box is the area covered by blocks 21, 22, 23 and 24 . The gray points represent the $\mathrm{K}$ giants located within $2 \mathrm{kpc}$ in the survey.

the survey around the Galactic longitude $l \sim 180^{\circ}$. Sixteen $10^{\circ} \times 10^{\circ}$ sky blocks are distributed around the anti-centre direction, and are numbered from 01 to 16 . Most contain a few thousand stars, except for blocks 04, 09, 13. In the area covered by $06,07,10,11,4$ other blocks cover the radial distance from $2 \mathrm{kpc}$ to $3 \mathrm{kpc}$. They are labelled as 17, 18, 19, 20. Two blocks, 21 and 22 , cover the even larger distances of 3-4 kpc and 4-5 kpc, respectively. Block 23 covers 5-7 kpc, and block 24 covers $7-12 \mathrm{kpc}$. The information is summa- rized in Table 1. The total number of selected $\mathrm{K}$ giants in the anti-centre direction is 47,461 . In addition, we use blocks 25 and 26 with $700 \mathrm{~K}$ giant stars to cover the north Galactic pole to $3 \mathrm{kpc}$ so that the kinematics in the vertical direction may be well detected. The $\mathrm{K}$ giants data sample includes sky positions, distances from the Sun, radial velocities and chemical information for all stars. 


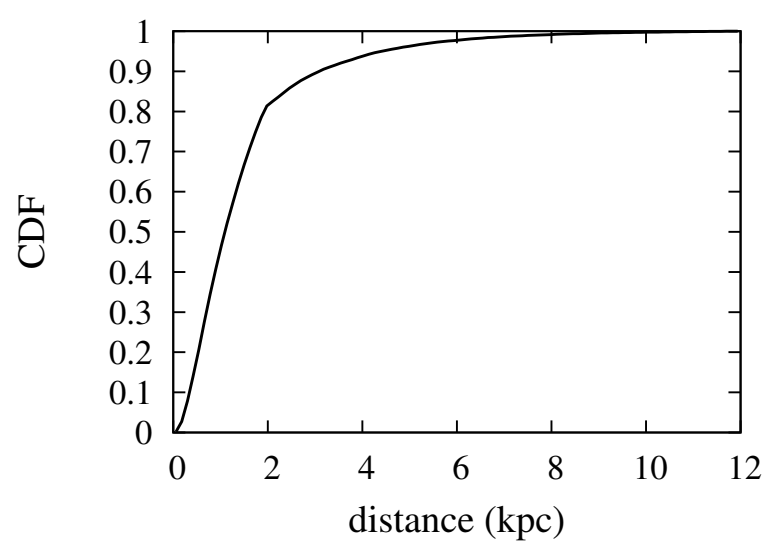

Figure 2. The cumulative distribution of $\mathrm{K}$ giants as a function of distance from the Sun. $20 \%$ of stars are outside of $\sim 2 \mathrm{kpc}$.

\section{TORUS MODEL \& GALACTIC MODEL}

We make no extensions to torus modelling theory nor to the Torus Mapper software. A fuller account of both may be found in Binney \& McMillan (2016) and Sanders \& Binney (2016).

\subsection{Action-angle variables}

In a static or quasi-static axisymmetric conservative potential, in addition to the total energy $E$ and the $z$ component of the angle momentum $L_{z}$, a third integral $I_{3}$ exists for regular stellar orbits. Since an orbit trajectory projected onto the meridional plane $\{R, z\}$ oscillates about a circular radius, the actions $J_{R}$ and $J_{z}$ in phase space $\{\boldsymbol{q}, \boldsymbol{p}\}$,

$J_{i}=\frac{1}{2 \pi} \oint_{\gamma_{i}} \boldsymbol{p} \cdot \mathbf{d} \boldsymbol{q}$,

replace $E$ and $I_{3}$ with the third action being $J_{\phi}=L_{z}$ (Arnold 1997; Sanders \& Binney 2016). Phase space is now described by the angle action variables $\{\boldsymbol{\theta}, \boldsymbol{J}\}$ rather than $\{\boldsymbol{q}, \boldsymbol{p}\}$. The Hamiltonian $H$ does not depend on the angles $\boldsymbol{\theta}$ and so $H \equiv H(\boldsymbol{J})$. The equations of motion of the stars are given by

$\dot{\boldsymbol{J}}=0, \quad \dot{\boldsymbol{\theta}}(t)=\frac{\partial H}{\partial \boldsymbol{J}}=\boldsymbol{\omega}(\boldsymbol{J})$.

It is clear that the angle variables $\boldsymbol{\theta}(t)$ can be written as

$\boldsymbol{\theta}(t)=\boldsymbol{\theta}(0)+\boldsymbol{\omega} t$.

Orbits in such a system can be expanded in complex variable terms as

$x(\boldsymbol{\theta}, \boldsymbol{J})=\sum_{\mathbf{k}} X_{\mathbf{k}}(\boldsymbol{J}) \exp (i \mathbf{k} \cdot \boldsymbol{\theta})$,

where the sum is over all vectors $\mathbf{k}$ with integer components. Combining equation (4) with equation (3), we find that the spatial coordinates are Fourier series in time, in which every frequency is the linear combination of three fundamental frequencies. In six-dimensional space, an orbit moves only in the three $\boldsymbol{\theta}$ directions, over a surface being topologically equivalent to a three-dimensional torus (3-torus) with the actions $\boldsymbol{J}$ serving to label the orbits.

Only for few specific potentials, such as the Stäckel and isochrone potentials, can we find the analytic formula for actions in all canonical coordinates. In order to describe the complicated mass model of our Galaxy, a procedure based on canonical transformations is developed for a general potential (McGill \& Binney 1990; Binney \& Kumar 1993). Firstly, a well-understood potential is employed as a reference (toy potential), to connect a point from the configuration velocity space $\{\boldsymbol{x}, \boldsymbol{v}\}$ to action-angle coordinates $\left\{\boldsymbol{\theta}^{T}, \boldsymbol{J}^{T}\right\}$, where the superscript $T$ represents toy. Then all we need to find is a relationship to connect the general potential to the toy potential. Since a canonical transformation keeps the topological structure, all we need is to find a generating function for the canonical transformation. Such a function is

$S\left(\boldsymbol{\theta}^{T}, \boldsymbol{J}\right)=\boldsymbol{\theta}^{T} \cdot \boldsymbol{J}-i \sum_{n \neq 0} S_{n}(\boldsymbol{J}) e^{i n \boldsymbol{\theta}^{T}}$,

in which the series of unknown terms $S_{n}$ (McGill \& Binney 1990) is to be determined. Note that $S_{n}$ are constants for a given $\boldsymbol{J}$. When we gain the approximation of this generating function, $\boldsymbol{J}^{T}$ can be simply obtained by the equation $\boldsymbol{J}^{T}=$ $\nabla_{\boldsymbol{\theta}^{T}} S\left(\boldsymbol{\theta}^{T}, \boldsymbol{J}\right)$. In this way, it is possible to map a point from $\{\boldsymbol{\theta}, \boldsymbol{J}\}$ through $\left\{\boldsymbol{\theta}^{T}, \boldsymbol{J}^{T}\right\}$, then to the configuration space $\{\boldsymbol{x}, \boldsymbol{v}\}$. The key point is to compute the coefficients of $S_{n}$. Instead of direct computation, a fitting method can estimate the $S_{n}$ by minimizing the error in the Hamilton resulting from the $S_{n}$ (McGill \& Binney 1990; Kaasalainen \& Binney 1994).

Such a procedure is complicated. In this work, we use a public package of routines Torus Mapper (Binney \& McMillan 2016) to map points from action-angle space to our more usual coordinates (see Section 4).

\subsection{Mass model and distribution function}

In this work, we assume that the Milky Way is axisymmetrical with a gas disc, two stellar discs, and a spheroidal halo and bulge. The density of discs is written as (Dehnen \& Binney 1998)

$\rho_{d}(R, z)=\frac{\Sigma_{d}}{2 z_{d}} \exp \left(-\frac{R}{R_{d}}-\frac{|z|}{z_{d}}-\frac{R_{h}}{R}\right)$

where $R_{d}$ is the scale length, $z_{d}$ is the scale height, and $\Sigma_{d}$ is the central surface density. The parameter $R_{h}$ describes a central depression, and is set to be non-zero for the gas disc, and zero for the stellar discs. The spheroidal components have the form

$\rho_{s}(R, z)=\frac{\rho_{0}}{m^{\gamma}(1+m)^{\beta-\gamma}} \exp \left[-\left(\frac{r_{0} m}{r_{\text {cut }}}\right)^{2}\right]$

where

$m(R, z)=\sqrt{\left(\frac{R}{r_{0}}\right)^{2}+\left(\frac{z}{q r_{0}}\right)^{2}}$,

and $\rho_{0}$ is the central density, $r_{0}$ is a scale radius and the parameter $q$ is the axial ratio of the isodensity surfaces. The parameters $\gamma$ and $\beta$ are the slopes for the inner and outer density profiles respectively, and $r_{c u t}$ is the cutoff radius. Most of our Milky Way models consist of a gas disc, two stellar discs, a halo and a bulge, except for M11b and M11c (See Table 3). It is clear that the potential of each disc component has 4 parameters, while each spheroidal component 
has 5 parameters $\left(\rho_{0}, \gamma, \beta, r_{0}, r_{c u t}\right)$. Therefore, we have 22 parameters in total for one MW density model. The main parameters are listed in Table 3.

Following Binney (2012), the distribution function of each single stellar disc can be assumed to be

$f\left(J_{R}, J_{z}, L_{z}\right)=\frac{\Omega \Sigma \nu}{2 \pi^{2} \sigma_{r}^{2} \sigma_{z}^{2} \kappa} \exp \left(-\frac{\kappa J_{R}}{\sigma_{r}^{2}}-\frac{\nu J_{z}}{\sigma_{z}^{2}}\right) T\left[\frac{L_{z}}{L_{0}}\right]$,

where $\Omega, \kappa$, and $\nu$ are the circular, radial, and vertical epicycle frequencies respectively. $\Sigma$ is the radial surface density profile. $T\left[L_{z} / L_{0}\right]$ is a function of $\left[1+\tanh \left(L_{z} / L_{0}\right)\right]$, with characteristic angular momentum $L_{0}$. All of them are functions of $L_{z}$. In this work, the total distribution function of actions is a combination of the thin and thick discs, their ratio is a free parameter with a default value of 0.7 .

The surface density of a disc is an exponential function

$\Sigma\left(L_{z}\right)=\Sigma_{0} \exp \left(-\frac{R_{c}}{R_{d}}\right)$,

where radius $R_{c}$ is derived by assuming a circular orbit with angular momentum $L_{z}$. Given the radius of the Solar circle $R_{0}$, the vertical and radial velocity dispersions are controlled by the scale parameter $R_{\sigma}$

$\sigma_{r}=\sigma_{r 0} \exp \left(\frac{R_{0}-R_{c}}{R_{\sigma}}\right), \sigma_{z}=\sigma_{z 0} \exp \left(\frac{R_{0}-R_{c}}{R_{\sigma}}\right)$.

The distribution function of a single disc is controlled by 4 parameters $\sigma_{r 0}, \sigma_{z 0}, R_{d}$, and $R_{\sigma}$. The $L_{0}$ truncation scale parameter is fixed at the Torus Mapper value of 9780 $\mathrm{kpc} \mathrm{km} \mathrm{s}{ }^{-1}$ (Binney \& McMillan 2016). An extra parameter is needed to adjust the ratio of the thick to thin discs. In total we have 9 free parameters to control the DF of our two component stellar disc system.

We build two sets of parameters for the motion of the Sun. Group 1 has $R_{0}=8.0 \mathrm{kpc}, V_{c}=220 \mathrm{~km} \mathrm{~s}^{-1}$, and a Solar motion of $(9.58,10.52,7.01) \mathrm{km} \mathrm{s}^{-1}$ with respect to the local standard of rest (LSR), as described in Tian et al. (2015). Group 2 has $R_{0}=8.5 \mathrm{kpc}, V_{c}=244.5 \mathrm{~km} \mathrm{~s}^{-1}$, and (11.1, 12.24, 7.25) $\mathrm{km} \mathrm{s}^{-1}$ (Schönrich et al. 2010; Piffl et al. 2014).

\subsection{Top level modelling process}

There are over 30 parameters in our models. This high number means that it is too expensive computationally to directly constrain all of these parameters simultaneously, even if an MCMC framework is used. As a consequence, we consider the parameters in two groups, those parameters affecting the mass models and those affecting the DFs. Our modelling procedure to find the best-fitting models and parameters matching our data is a two step procedure using these two groups. We assess the fit of our models to the data using a least $\chi^{2}$ approach (see below).

In the first step of our procedure, mass models are examined with fixed DF parameters. Because of the number of mass parameters, 36 mass models are adopted to cover the parameter space. Given an initial set of DF parameters, a best-fitting mass model is found. For the second step, we then adjust the DF parameters one by one based on the current best-fitting mass model. If the new DF parameters support a different mass model (having a smaller $\chi^{2}$ value), then this mass model is set as the new reference. We iterate the two-step process until we find the best-fitting parameters for both the mass and DF parts of our model.

The definition of $\chi^{2}$ we use for a single data block is

$\chi^{2}=\sum_{n} \frac{\left(p_{n}^{\text {data }}-p_{n}^{\text {th }}\right)^{2}}{\sigma_{n}^{2}}$,

where $p_{n}^{\text {data }}$ is the line-of-sight velocity distribution with Poisson noise $\sigma_{n}$ constructed from our LAMOST data, and $p_{n}^{\text {th }}$ is the model prediction. The overall $\chi^{2}$ value for model comparison purposes is simply the sum of the individual block $\chi^{2}$ values. For each block, we compare the model with the data at 40 points. For our 26 data blocks, the total number of points is $n=26 \times 40$, and the degrees of freedom value (d.o.f) is $n-32=1008$. We ignore any correlation between blocks.

Our data line-of-sight velocity distribution for a block is formed by binning the $\mathrm{K}$ giant radial velocity values to give a data distribution histogram. The model line-of-sight velocity distribution is formed using Torus Mapper to create a set of model $\mathrm{K}$ giants which are then binned by velocity to give the model distribution histogram.

Torus Mapper (TM, Binney \& McMillan 2016) is an object-oriented $\mathrm{C}++$ toolkit, which provides a user-friendly interface for generating tori. We use its AutoFit routine to build torus libraries. For each model, a Monte Carlo Markov Chain (MCMC) sampler generates over 2 million points in action space, using our DF parameters and the parameters of our mass model. Every point is taken to represent a model K giant. Next we use FullMap to map the stars from action angle variables to configuration space, and the line-of-sight velocity distribution is constructed from the model stars as above. We have tried modelling with more than 2 million points and rarely find that the modelling accuracy improves, only that modelling becomes more time-consuming. As a guideline, a single, typical model costs roughly $20 \mathrm{cpu}$ hours to produce.

\section{RESULTS}

\subsection{Consistent results in the solar vicinity}

Our current observations provide a constraint for the solar neighbourhood. We check whether our LAMOST data are consistent with the conventional understanding of the solar vicinity, e.g. as in Binney (2012) from which we use its first potential and corresponding DF parameters as a reference model. Density parameters are listed in Table 3 and DF parameters can be found found in the first row of Table 2 . In what follows, this group of parameter is referred to as B12. It is apparent that LAMOST data are consistent with the B12 model for the nearby blocks 1-16 and 25-26. All of these blocks are located within $\sim 2 \mathrm{kpc}$. This demonstrates that the LAMOST data are consistent with the previous best understanding of kinematics in the solar vicinity. Note that we cannot completely follow the original B12 model due to limitations in the public interface of TM. The DF of B12 can be a superposition of multiple quasi-isothermal components, but we only use fixed thin/thick components because of the TM software interface. We have compared 


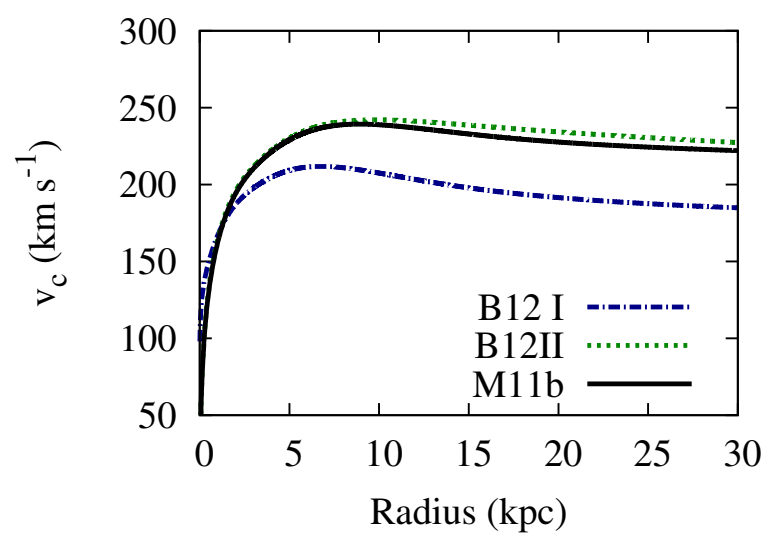

Figure 3. Rotation curves for the 3 main mass models. M11b (black solid curve) is the potential of the best-fitting model, and the reference potentials are B12 II (green dot-dashed curve) and B12 I (blue dotted curve).

DFs from our procedure and B12, and the amplitude and trends are consistent with each other.

We illustrate the data l.o.s velocity distribution and our fiducial model. Fig. 4 shows that the velocity distributions of block $01-16$ are well matched with the models. The results for blocks $17-24$, plotted in Fig. 5, correspond to more distant sky regions. The results for blocks 25 and 26, plotted in Fig. 6, correspond to the northern Galactic pole.

Since B12 is constrained only by data within $\sim 2 \mathrm{kpc}$, e.g. GCS, we found that the natural extension of B12 beyond the solar vicinity cannot match the data in the outer region. As shown in Fig. 5, B12 predicts a relatively small velocity dispersion and the deviation increases as the radius increases. In the next section, we change our parameters to seek a more general model to explain the outer region.

\subsection{The hot outer disc}

In order to find our best-fitting model, we experiment with parameters for density and DF across a wide range. Given the thin/thick decomposition, we show the $\chi^{2}$ values for different models. We start with an initial set of DF parameters based on Binney (2012) and Piffl et al. (2014). We change iteratively the parameters and find our fiducial DF parameters, those which give the least $\chi^{2}$ values within $10 \%$ uncertainty of the current best-fitting model. The DF parameters are listed in the first row of Table 2. Our best-fitting DF parameters are listed in Table 2 and labelled as 'fid' and with $\chi^{2}=2.08$. This model does describe the hot disc and behaves much better than B12 in the outer disc. However, the velocity dispersion $\sigma_{z 0}$ of the thick disc is $\sim 80 \mathrm{~km} \mathrm{~s}^{-1}$ and the scale length for velocity dispersion $R_{\sigma}$ is close to 20 kpc. This suggests the existence of a hot, extended thick disc reaching much beyond the solar neighborhood. The $R_{\sigma}$ parameter is usually set to be twice the scale length (van der Kruit \& Searle 1981; Lewis \& Freeman 1989). In this work, we set it to be free. Liu et al. (2017a) found from the radial stellar surface density profile measured with the LAMOST giant stars that the outer disc also extends as far as $19 \mathrm{kpc}$ in Galactocentric radius. This could be associated with the large $R_{\sigma}$ suggested by the best-fitting model.
Furthermore, we examine the effect with the DF parameters. Around the fiducial parameters, the scale length $R_{d}$ and dispersion $R_{\sigma}$, velocity dispersion $\sigma_{(r, z)}$ and the ratio between the two discs are considered. We estimate $\chi^{2}$ for four block ranges. The first range consists of block $\{01-16\}$ and roughly corresponds to the range of RAVE data. Its $\chi^{2}$ value is referred to as $\chi_{\text {inner }}^{2}$. For the middle blocks, $\chi_{\text {middle }}^{2}$ is for blocks $\{17-20\}$, and $\chi_{\text {outer }}^{2}$ is for blocks $\{23,24\}$, which cover the outer region of the MW. To verify the robustness of our best-fitting parameters as a fiducial model, ten models are created around the best-fitting point in parameter space. We find that $\chi^{2}$ increases quickly when $\sigma_{z 0}$ decreases to $\sim 70 \mathrm{~km} \mathrm{~s}^{-1}$ (model 3 in Table 2). Thus the fiducial model appears to be robust.

Given the fiducial DF parameters (first row in Table 2), we examine 36 mass models of the MW. The notations of P14, B12I, B12II and BT08 represent the parameters from Piffl et al. (2014), Binney (2012) and Binney \& Tremaine (2008), respectively. M11b denotes the best model and M11c denotes the convenient model from McMillan (2011). The other mass models are from Dehnen \& Binney (1998). The results are listed in Table 3. The solid curve in the DF Figure is the best-fitting model. Any deviations become more apparent in Figs. 5 and 6 .

The $\chi^{2}$ values of the Group 2 models are in general smaller than Group 1. This implies that the solar motion or position in Group 1 may have been systematically underestimated. In what follows, we only show the results for Group 2.

Besides the DF parameters, the mass models also favour a large disc. The mass models with larger $R_{d}$, such as 40 , $2 \mathrm{~b}, 2 \mathrm{~d}$ and $4 \mathrm{~d}$ et c., tend to have smaller $\chi^{2}$ values. More sophisticated mass models will be considered in a future work.

\subsection{Tension between the models and data}

From the analysis in the last section, our fiducial parameters can roughly match the $\mathrm{DF}$, but the two wings of the l.o.s velocity DF in blocks 25 and 26 do not show the characteristics of halo stars (See Fig. 5). On the other hand, B12 can explain the RAVE data at the Galactic pole better than our model (see Fig. 6). Our preferred model gives a larger velocity dispersion than the block 25 data. However, we cannot find a model to match both sides under the assumption of a two component decomposition. An improvement in the theory is required. The simplest way is to add an extra component for the outer region beyond the solar vicinity.

Since the scale radius and velocity dispersion of the thick disc are both larger than the thin disc, the long thick disc is more like an envelope of the short, thin disc in the MW outskirts. Thus we add the third disc based around the fiducial parameters, e.g. $\sigma_{z} \sim 80 \mathrm{kms}^{-1}$. Around these initial parameters, we run several dozens of models to find a 'better' model. We find that some models can fit (with $\left.\chi^{2} \leqslant 2\right)$ the outer data, at about $(15,20) \mathrm{kpc}$, but fit poorly $\left(\chi^{2} \geqslant 4\right)$ the intermediate region about $(12,15) \mathrm{kpc}$ and vice versa. Because we control the mass fraction of the third component to below 0.3 to hold a relatively small $\sigma_{z}$ in the Solar vicinity, the intermediate region is not dominated by the third disc and it only has a significant effect in the outermost region. 
Table 2. $\chi^{2}$ for the best-fitting parameters of the distribution functions. The fiducial parameters of the DF are labeled by fid. The row of B12 corresponds to the parameters in Binney (2012). The rows labelled from $1-10$ are the models around the second row ( $f i d$, M11b) $\cdot \chi_{\text {inner }}^{2}$ is estimated by the first 16 blocks in the Solar neighbourhood, $\chi_{\text {middle }}^{2}$ by $(17-20), \chi_{\text {outer }}^{2}$ by $(23,24), \chi_{\text {Pole }}^{2}$ by $(25,26)$ and $\chi_{\text {Total }}^{2}$ is estimated by all of 26 blocks (the blocks are defined in Table 1 ).

\begin{tabular}{|c|c|c|c|c|c|c|c|c|c|c|c|c|c|c|}
\hline & \multicolumn{4}{|c|}{ Thin } & \multicolumn{4}{|c|}{ Thick } & \multirow[t]{2}{*}{ ratio } & \multicolumn{5}{|c|}{$\chi^{2} /$ d.o.f } \\
\hline & 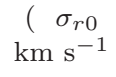 & $\begin{array}{c}\sigma_{z 0} \\
\mathrm{~km} \mathrm{~s}^{-1}\end{array}$ & $\begin{array}{c}R_{d} \\
\mathrm{kpc}\end{array}$ & $\begin{array}{l}\left.R_{\sigma}\right) \\
\mathrm{kpc}\end{array}$ & 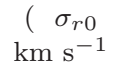 & $\begin{array}{c}\sigma_{z 0} \\
\mathrm{~km} \mathrm{~s}^{-1}\end{array}$ & $\begin{array}{c}R_{d} \\
\mathrm{kpc}\end{array}$ & $\begin{array}{c}\left.R_{\sigma} \quad\right) \\
\mathrm{kpc}\end{array}$ & & inner & $\begin{array}{l}\text { anti-centre } \\
\text { middle }\end{array}$ & outer & Pole & Total ) \\
\hline B12 & 40.1 & 25.6 & 2.58 & 8.93 & 25.8 & 45.0 & 2.11 & 4.04 & 0.772 & 3.01 & 4.28 & 32.1 & 1.13 & 4.50 \\
\hline fid & 29.0 & 42.9 & 2.41 & 10.8 & 50.6 & 79.3 & 4.07 & 19.3 & 0.67 & 2.27 & 2.53 & 2.38 & 1.85 & 2.08 \\
\hline 1 & 26.1 & 42.9 & 2.41 & 10.8 & 45.5 & 79.3 & 4.07 & 19.3 & 0.67 & 2.67 & 3.54 & 4.85 & 2.26 & 2.61 \\
\hline 2 & 31.9 & 42.9 & 2.41 & 10.8 & 55.7 & 79.3 & 4.07 & 19.3 & 0.67 & 3.35 & 2.42 & 5.04 & 3.77 & 2.94 \\
\hline 4 & 29.0 & 47.2 & 2.41 & 10.8 & 50.6 & 87.2 & 4.07 & 19.3 & 0.67 & 2.32 & 2.50 & 5.36 & 2.17 & 2.25 \\
\hline 5 & 29.0 & 42.9 & 2.17 & 10.8 & 50.6 & 79.3 & 3.66 & 19.3 & 0.67 & 2.52 & 2.56 & 3.62 & 3.64 & 2.37 \\
\hline 6 & 29.0 & 42.9 & 2.65 & 10.8 & 50.6 & 79.3 & 4.48 & 19.3 & 0.67 & 2.39 & 2.89 & 2.91 & 2.58 & 2.26 \\
\hline 7 & 29.0 & 42.9 & 2.41 & 9.72 & 50.6 & 79.3 & 4.07 & 17.4 & 0.67 & 2.21 & 2.88 & 2.81 & 2.50 & 2.15 \\
\hline 8 & 29.0 & 42.9 & 2.41 & 11.9 & 50.6 & 79.3 & 4.07 & 21.2 & 0.67 & 2.47 & 2.82 & 4.34 & 3.34 & 2.39 \\
\hline 9 & 29.0 & 42.9 & 2.41 & 10.8 & 50.6 & 79.3 & 4.07 & 19.3 & 0.60 & 2.27 & 2.81 & 4.16 & 3.43 & 2.28 \\
\hline 10 & 29.0 & 42.9 & 2.41 & 10.8 & 50.6 & 79.3 & 4.07 & 19.3 & 0.74 & 2.39 & 2.37 & 5.96 & 2.42 & 2.32 \\
\hline
\end{tabular}

The thin/thick disc decomposition is well known for the solar vicinity, but the parameters and cause of the thick disc (geometrical, kinematical, and chemical and so on) are unclear. A straightforward idea is that the thick disc is formed from the heating of old stars while young stars are in the thin disc. Therefore the geometrically-thick disc should correspond to the old population. Current observations indicate that there is a radial age gradient (Martig et al. 2016), corresponding to a complex kinematic structure. However, for discs, a possible flaring structure can also exist in the disc outskirts (Bovy \& Rix 2013; Bovy et al. 2016).

The number of $\mathrm{K}$ giants in this work is insufficient to tell which kinds of structures are required. The previous thin/thick decomposition is however rejected as are the modified (our fiducial) 2-disc model, and the 3-disc model. If we introduce more components with different scale lengths and velocity dispersions, perhaps a solution for the whole region can be developed, but it would require a complex form of DF. In summary, our current torus model still needs to be developed further theoretically.

\section{DISCUSSION AND SUMMARY}

We have successfully met our aims as set out in the Introduction. Our results corroborate earlier results and we have produced a model for the Milky Way's outer disc.

It is the first time the torus approach has been applied to the LAMOST survey. The torus mapper package TM (Binney \& McMillan 2016) has been employed to construct our torus models for different density models with different DF parameters. The data are well matched by the model with the mass model of M11b and by our fiducial DF parameters (the first row in Table 2), except for the Solar neighborhood. We are forced to have a thick disc that is much more extended and thicker than previously thought. If such a component exists, more careful analyses of age and metallicity information should yield more solid evidence. Given the quasi-thermal disc assumption, there must be a sharp transition of the thin/thick model from the solar vicinity to the outer region. We also test whether a simple additional disc can match the data. The answer is in the negative, which suggests that a more complex DF is needed to accommodate the outer l.o.s velocity distribution as revealed by the LAMOST K giant data.

The advantage of LAMOST data is its range, which allows us to study the stellar distribution over $2 \mathrm{kpc}$. It has helped to rule out some mass models, such as mass model numbers 10 or 20 (see Table 3 ). However the tangential velocities of those distant stars are unconstrained. It is still insufficient to completely break the degeneracy between individual components or parameters within the mass models. Meanwhile, the information on age and metallicity needs to be taken into account in future models.

Although the spatial distribution of stars in block 9 is extremely inhomogeneous, it does not introduce any apparent bias in the DF. The non-uniformity of spatial distribution in a block is not a serious problem in our work. There are differences in many blocks, such as blocks 07, 08, 15, 18 and 20. The reasons may be complex. It could imply some distortion of the MW disc, but our theory is based on a symmetric potential and simple DF model. Sub-structures and bulk motion can also change the shape of the l.o.s velocity DF.

Finally, the axisymmetry assumption in the torus model may be over-simplified. In fact, many observations indicate that neither the disc star counts nor the disc stellar kinematics are axisymmetric. Widrow et al. (2012) and Xu et al. (2015) claimed that the stellar density of the disc shows quite complicated vertical oscillations in the solar neighborhood and the disc outskirts, respectively. Meanwhile, Williams et al. (2013) and Carlin et al. (2013) found that the stars have bulk motions in both the radial and vertical directions in the solar vicinity. Also, Liu et al. (2017b) demonstrated that the azimuthal velocity also contains different asymmetric components for stars with different ages within 600 pc around the Sun. Moreover, Tian et al. (2016) identified asymmetric radial and azimuthal velocities from $R \sim 8.5$ to $14 \mathrm{kpc}$. These peculiar velocities located across the radial range of the outer disc may potentially affect the 


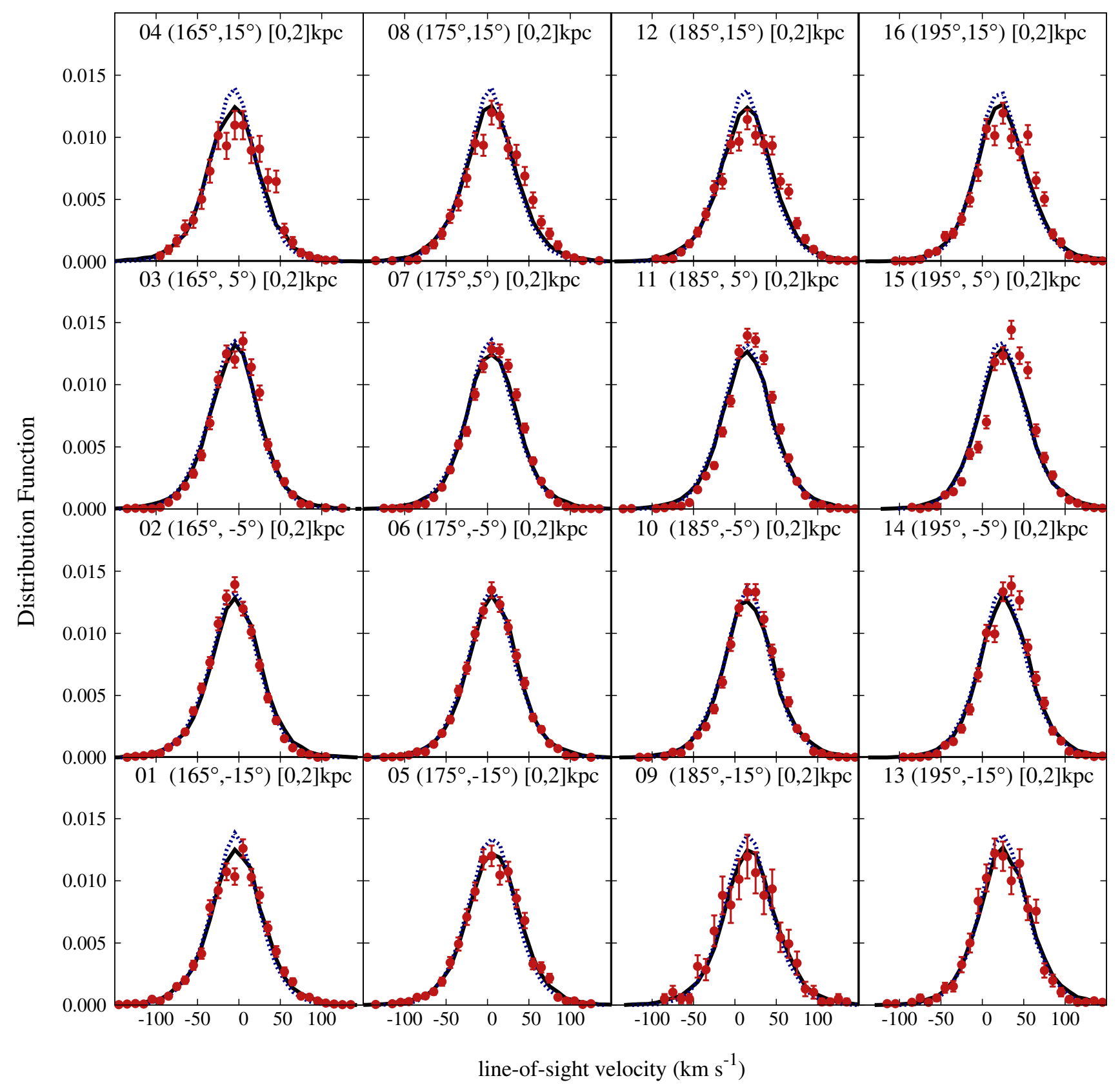

Figure 4. The probability distribution function of line-of-sight velocity for blocks $01-16$. The upper left number in each panel is the rank of the block. The corresponding distance range is listed in Table 1. The black solid and the blue dotted curves represent the predictions from M11b, and B12, respectively. The red points denote the data together with their Poisson error bars. Each block is $10^{\circ} \times 10^{\circ}$. The longitude and latitude of each block centre is indicated together with distance range.

comparison between the oversimplified torus model and the observed data. Indeed, Fig. 5 does show that the observed l.o.s. velocity distributions are slightly asymmetric. However, the asymmetry in these distributions is mostly less than $10 \mathrm{~km} \mathrm{~s}^{-1}$, which should not substantially affect fitting a model since the velocity dispersion values are much larger than the asymmetric velocity by a factor of a few $(<10)$.

To summarise, we have built a model of the Milky Ways's outer disc but it shows that a more sophisticated distribution function is required to model the observed velocity distribution.

\section{ACKNOWLEDGEMENTS}

We thank James Binney and the anonymous referee for their very useful comments and corrections. We acknowledge support from the National Natural Science Foundation of China (Grant No. 11333003, 11373032, 11390372, 11403035), and 


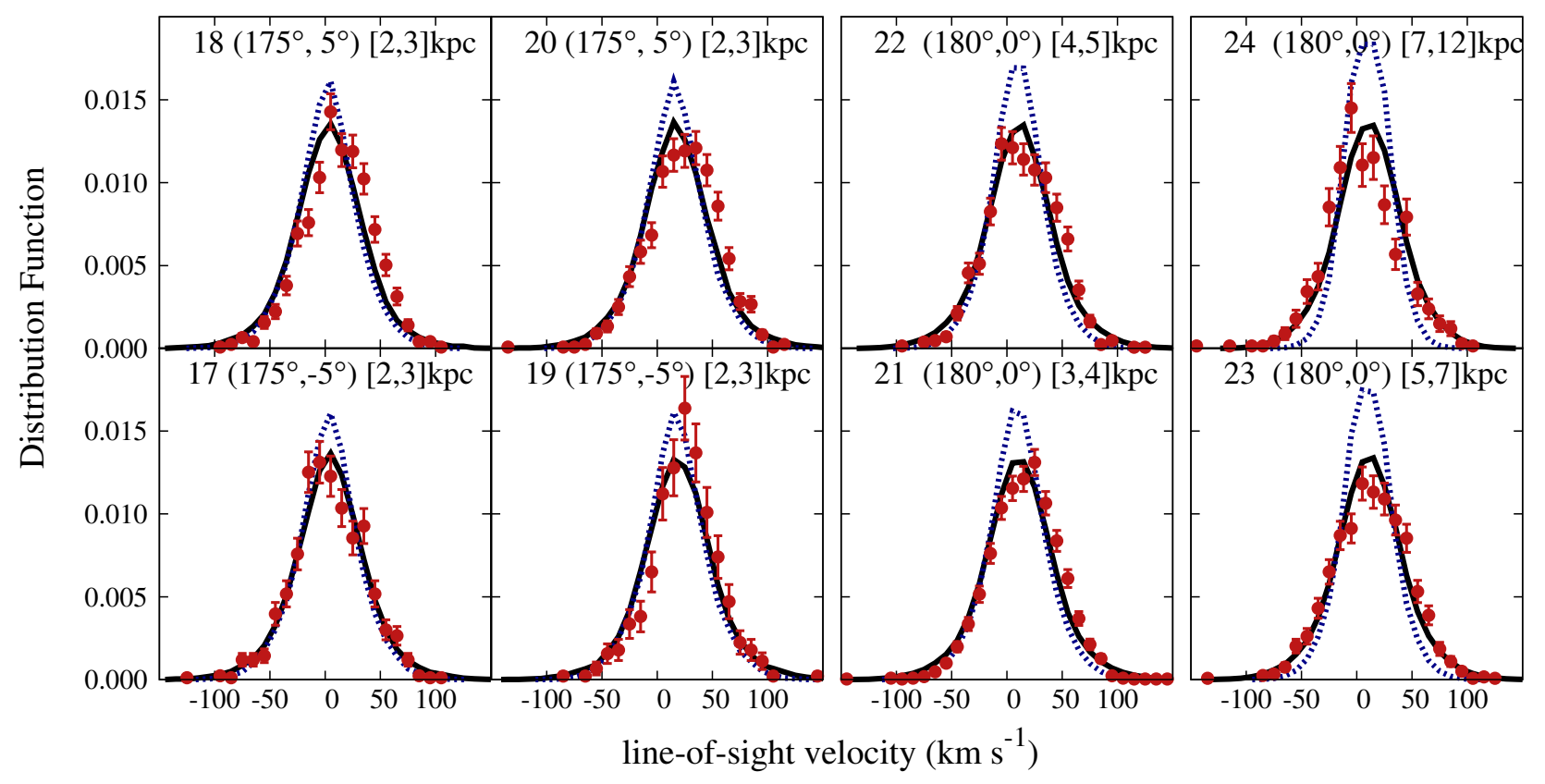

Figure 5. The probability distribution function of the line-of-sight velocity for blocks $17-24$. The upper left number in each panel is the rank of the block. The corresponding range is listed in Table 1. The black solid and the blue dotted curves represent the predictions from M11b and B12, respectively. The red points denote the data together with the Poisson errors. The longitude and latitude of the centre of each block is indicated together with distance range. Blocks $21-24$ are $20^{\circ} \times 20^{\circ}$.

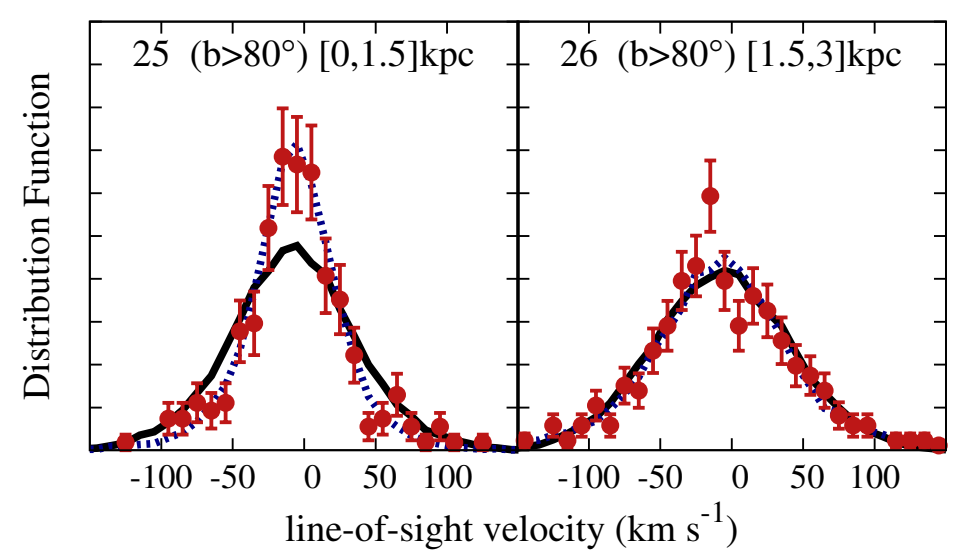

Figure 6. The probability distribution density function of the line-of-sight velocity for blocks 25 (left) and 26 (right). The corresponding range is listed in Table 1. The black solid and the blue dotted curves represent the predictions from M11b and B12, respectively. The red points denote the data together with the Poisson errors. The latitude range is indicated together with distance range. The two blocks cover an area of $10^{\circ} \times 10^{\circ}$ in the north Galactic pole direction.

from the 973 Program (No. 2014CB845700). This work is also supported by the Strategic Priority Research Program "The Emergence of Cosmological Structures" of the Chinese Academy of Sciences (Grant No. XDB09000000). The Guoshoujing Telescope (the Large Sky Area Multi-Object Fiber Spectroscopic Telescope LAMOST) is a National Major Scientific Project built by the Chinese Academy of Sciences. Funding for the project has been provided by the National Development and Reform Commission. LAMOST is operated and managed by the National Astronomical Observatories, Chinese Academy of Sciences.

\section{REFERENCES}

Allende Prieto C., et al., 2008, Astronomische Nachrichten, 329, 1018

Arnold V. I., 1997, Mathematical Methods of Classical Mechanics: Second Edition. Springer

Astraatmadja T. L., Bailer-Jones C. A. L., 2016, ApJ, 833, 119

Binney J., 2012, MNRAS, 426, 1328

Binney J., Kumar S., 1993, MNRAS, 261, 584

Binney J., McMillan P. J., 2016, MNRAS, 456, 1982

Binney J., Piff T., 2015, MNRAS, 454, 3653

Binney J., Tremaine S., 2008, Galactic Dynamics: Second Edition. Princeton University Press

Binney J., et al., 2014, MNRAS, 439, 1231 
Table 3. The reduced $\chi^{2}$ for different mass models. The main parameters of discs, bulge and dark halo are listed in the table. G1 and G2 correspond to the two groups with differing solar position and motions. The best-fitting model is M11b in Group 2. The notation of different mass models is described in Sec. 4.

\begin{tabular}{|c|c|c|c|c|c|c|c|c|c|c|c|c|c|c|}
\hline & \multicolumn{2}{|c|}{ Thin } & \multicolumn{2}{|c|}{ Thick } & \multicolumn{2}{|c|}{ Gas } & \multicolumn{3}{|c|}{ bulge } & \multicolumn{3}{|c|}{ halo } & \multicolumn{2}{|c|}{$\chi^{2} /$ d.o.f } \\
\hline & $\begin{array}{c}\left(\Sigma_{0}\right. \\
\mathrm{M}_{\odot} / \mathrm{kpc}^{2}\end{array}$ & $\begin{array}{l}\left.R_{d}\right) \\
\mathrm{kpc}\end{array}$ & $\begin{array}{c}\left(\Sigma_{0}\right. \\
\mathrm{M}_{\odot} / \mathrm{kpc}^{2}\end{array}$ & $\begin{array}{l}\left.R_{d}\right) \\
\mathrm{kpc}\end{array}$ & $\begin{array}{c}\left(\Sigma_{0}\right. \\
\mathrm{M}_{\odot} / \mathrm{kpc}^{3}\end{array}$ & $\begin{array}{l}\left.R_{d}\right) \\
\mathrm{kpc}\end{array}$ & $\begin{array}{c}\left(\rho_{0}\right. \\
\mathrm{M}_{\odot} / \mathrm{kpc}^{3}\end{array}$ & $q$ & $\begin{array}{l}\left.r_{0}\right) \\
\mathrm{kpc}\end{array}$ & $\begin{array}{c}\left(\rho_{0}\right. \\
\mathrm{M}_{\odot} / \mathrm{kpc}^{3}\end{array}$ & $q$ & $\left.r_{0}\right)$ & G1 & G2 \\
\hline P14 & $5.71 \mathrm{e} 8$ & 2.68 & $2.51 \mathrm{e} 8$ & 2.68 & $9.45 \mathrm{e} 7$ & 5.36 & $9.49 \mathrm{e} 10$ & 0.5 & 0.075 & $1.81 \mathrm{e} 7$ & 1 & 14.4 & 4.32 & 2.59 \\
\hline B12I & $1.02 \mathrm{e} 9$ & 2.4 & $1.14 \mathrm{e} 6$ & 2.4 & $7.30 \mathrm{e} 7$ & 4.8 & $1.26 \mathrm{e} 9$ & 0.8 & 1.09 & $7.56 \mathrm{e} 8$ & 0.6 & 1 & 2.53 & 2.88 \\
\hline B12II & $7.68 \mathrm{e} 8$ & 2.64 & $2.01 \mathrm{e} 8$ & 2.97 & $1.16 \mathrm{e} 8$ & 5.28 & $9.49 \mathrm{e} 10$ & 0.5 & 0.075 & $1.32 \mathrm{e} 7$ & 1 & 16.5 & 4.27 & 2.54 \\
\hline M11c & $7.53 \mathrm{e} 8$ & 3.0 & $1.82 \mathrm{e} 8$ & 3.5 & - & - & $9.41 \mathrm{e} 10$ & 0.5 & 0.075 & $1.25 \mathrm{e} 7$ & 1 & 17 & 4.36 & 2.29 \\
\hline M11b & $8.17 \mathrm{e} 8$ & 2.9 & $2.09 \mathrm{e} 8$ & 3.31 & - & - & $9.56 \mathrm{e} 10$ & 0.5 & 0.075 & $8.46 \mathrm{e} 6$ & 1 & 20.2 & 3.73 & 2.08 \\
\hline ВT08 & $1.18 \mathrm{e} 8$ & 2.0 & $1.66 \mathrm{e} 9$ & 2.0 & $1.32 \mathrm{e} 8$ & 4.0 & $7.11 \mathrm{e} 8$ & 0.8 & 3.83 & $4.27 \mathrm{e} 8$ & 0.6 & 1 & 2.80 & 2.37 \\
\hline 1 & $1.65 \mathrm{e} 9$ & 2.0 & $1.32 \mathrm{e} 8$ & 4.0 & $1.18 \mathrm{e} 8$ & 2.0 & $7.11 \mathrm{e} 8$ & 0.8 & 3.83 & $4.27 \mathrm{e} 8$ & 0.6 & 1 & 3.14 & 2.51 \\
\hline 10 & $1.59 \mathrm{e} 9$ & 2.0 & $1.27 \mathrm{e} 8$ & 4.0 & $1.13 \mathrm{e} 8$ & 2.0 & $5.0 \mathrm{e} 8$ & 0.8 & 5.0 & $5.0 \mathrm{e} 8$ & 0.6 & 1 & 12.1 & 6.52 \\
\hline 2 & $1.02 \mathrm{e} 9$ & 2.4 & $1.14 \mathrm{e} 8$ & 4.8 & $7.30 \mathrm{e} 7$ & 2.4 & $1.26 \mathrm{e} 9$ & 0.8 & 1.09 & $7.56 \mathrm{e} 8$ & 0.6 & 1 & 3.08 & 2.81 \\
\hline 20 & $1.03 \mathrm{e} 9$ & 2.4 & $1.14 \mathrm{e} 8$ & 4.8 & $7.32 \mathrm{e} 7$ & 2.4 & $1.0 \mathrm{e} 9$ & 0.8 & 5.0 & $6.0 \mathrm{e} 8$ & 0.6 & 1 & 41.6 & 28.5 \\
\hline 2.1 & $1.05 \mathrm{e} 9$ & 2.4 & $1.17 \mathrm{e} 8$ & 4.8 & $7.52 \mathrm{e} 7$ & 2.4 & $1.06 \mathrm{e} 9$ & 0.9 & 1.19 & $7.54 \mathrm{e} 8$ & 0.6 & 1 & 3.18 & 2.58 \\
\hline 2.2 & $9.82 \mathrm{e} 8$ & 2.4 & $1.09 \mathrm{e} 8$ & 4.8 & $7.0 \mathrm{e} 7$ & 2.4 & $1.58 \mathrm{e} 9$ & 0.7 & 1.0 & $7.62 \mathrm{e} 8$ & 0.6 & 1 & 3.16 & 2.58 \\
\hline 2.3 & $9.19 \mathrm{e} 8$ & 2.4 & $1.02 \mathrm{e} 8$ & 4.8 & $6.57 \mathrm{e} 7$ & 2.4 & $1.70 \mathrm{e} 9$ & 0.6 & 1.0 & $7.59 \mathrm{e} 8$ & 0.6 & 1 & 3.19 & 2.58 \\
\hline 2.4 & $8.27 \mathrm{e} 8$ & 2.4 & $9.20 \mathrm{e} 7$ & 4.8 & $5.9 \mathrm{e} 7$ & 2.4 & $1.84 \mathrm{e} 9$ & 0.5 & 1.0 & $7.29 \mathrm{e} 8$ & 0.6 & 1 & 2.76 & 2.62 \\
\hline $2 \mathrm{a}$ & $1.03 \mathrm{e} 9$ & 2.25 & $1.18 \mathrm{e} 8$ & 4.5 & $7.36 \mathrm{e} 7$ & 2.25 & $9.17 \mathrm{e} 5$ & 0.8 & 22.8 & $3.96 \mathrm{e} 8$ & 0.6 & 1 & 2.70 & 3.49 \\
\hline $2 b$ & $9.87 \mathrm{e} 8$ & 2.55 & $1.07 \mathrm{e} 8$ & 5.1 & $7.06 \mathrm{e} 7$ & 2.55 & $1.42 \mathrm{e} 9$ & 0.8 & 1.73 & $7.87 \mathrm{e} 8$ & 0.6 & 1 & 3.60 & 2.21 \\
\hline $2 \mathrm{c}$ & $1.03 \mathrm{e} 9$ & 2.4 & $1.14 \mathrm{e} 8$ & 4.8 & $7.33 \mathrm{e} 7$ & 2.4 & $3.05 \mathrm{e} 7$ & 0.8 & 6.19 & $5.99 \mathrm{e} 8$ & 0.6 & 1 & 3.15 & 2.71 \\
\hline $2 \mathrm{~d}$ & $1.05 \mathrm{e} 8$ & 2.4 & $1.16 \mathrm{e} 8$ & 4.8 & $7.48 \mathrm{e} 7$ & 2.4 & $6.16 \mathrm{e} 7$ & 0.8 & 21.8 & $6.75 \mathrm{e} 8$ & 0.6 & 1 & 2.79 & 2.77 \\
\hline $2 \mathrm{e}$ & $1.07 \mathrm{e} 9$ & 2.4 & $1.09 \mathrm{e} 8$ & 4.8 & $7.68 \mathrm{e} 7$ & 2.4 & $9.08 \mathrm{e} 8$ & 0.8 & 1.80 & $6.58 \mathrm{e} 8$ & 0.6 & 1 & 3.08 & 2.37 \\
\hline $2 \mathrm{f}$ & $9.40 \mathrm{e} 8$ & 2.4 & $1.14 \mathrm{e} 8$ & 4.8 & $6.71 \mathrm{e} 7$ & 2.4 & $4.17 \mathrm{e} 8$ & 0.8 & 1.0 & $3.0 \mathrm{e} 8$ & 0.6 & 1 & 3.05 & 2.77 \\
\hline $2 \mathrm{~g}$ & $1.01 \mathrm{e} 9$ & 2.4 & $1.12 \mathrm{e} 8$ & 4.8 & $7.21 \mathrm{e} 7$ & 2.4 & $8.33 \mathrm{e} 8$ & 0.8 & 1.92 & $9.53 \mathrm{e} 8$ & 0.6 & 1 & 2.84 & 2.77 \\
\hline $2 \mathrm{~h}$ & $8.66 \mathrm{e} 8$ & 2.4 & $9.63 \mathrm{e} 7$ & 4.8 & $6.19 \mathrm{e} 7$ & 2.4 & $1.07 \mathrm{e} 9$ & 0.8 & 2.88 & $1.23 \mathrm{e} 9$ & 0.6 & 1 & 3.06 & 2.28 \\
\hline $2 \mathrm{i}$ & $7.85 \mathrm{e} 8$ & 2.4 & $8.73 \mathrm{e} 7$ & 4.8 & $5.6 \mathrm{e} 7$ & 2.4 & $8.51 \mathrm{e} 8$ & 0.3 & 1.0 & $3.38 \mathrm{e} 8$ & 0.6 & 1 & 2.78 & 3.04 \\
\hline $2 \mathrm{~L}$ & $1.02 \mathrm{e} 9$ & 2.4 & $1.13 \mathrm{e} 8$ & 4.8 & $7.3 \mathrm{e} 7$ & 2.4 & $1.66 \mathrm{e} 9$ & 0.8 & 1.09 & $7.56 \mathrm{e} 8$ & 0.6 & 1 & 3.49 & 2.53 \\
\hline $2 \mathrm{~S}$ & $1.02 \mathrm{e} 9$ & 2.4 & $1.13 \mathrm{e} 8$ & 4.8 & $7.30 \mathrm{e} 7$ & 2.4 & $9.13 \mathrm{e} 8$ & 0.8 & 1.09 & $7.56 \mathrm{e} 8$ & 0.6 & 1 & 2.84 & 3.37 \\
\hline 3 & $6.42 \mathrm{e} 8$ & 2.8 & $9.06 \mathrm{e} 7$ & 5.6 & $4.59 \mathrm{e} 7$ & 2.8 & $1.18 \mathrm{e} 8$ & 0.8 & 2.29 & $3.0 \mathrm{e} 8$ & 0.6 & 1 & 2.87 & 2.46 \\
\hline 4 & $4.32 \mathrm{e} 8$ & 3.2 & $7.28 \mathrm{e} 7$ & 6.4 & $3.09 \mathrm{e} 7$ & 3.2 & $2.66 \mathrm{e} 8$ & 0.8 & 1.90 & $3.0 \mathrm{e} 8$ & 0.6 & 1 & 3.05 & 2.50 \\
\hline 40 & $4.26 \mathrm{e} 8$ & 3.2 & $7.18 \mathrm{e} 7$ & 6.4 & $3.04 \mathrm{e} 7$ & 3.2 & $2.20 \mathrm{e} 8$ & 0.8 & 2.77 & $3.0 \mathrm{e} 8$ & 0.6 & 1 & 3.26 & 2.18 \\
\hline 4.1 & $4.52 \mathrm{e} 8$ & 3.2 & $7.63 \mathrm{e} 7$ & 6.4 & $3.23 \mathrm{e} 7$ & 3.2 & $2.24 \mathrm{e} 8$ & 0.9 & 1.93 & $3.0 \mathrm{e} 8$ & 0.6 & 1 & 3.01 & 2.48 \\
\hline 4.2 & $4.05 \mathrm{e} 8$ & 3.2 & $6.84 \mathrm{e} 7$ & 6.4 & $2.89 \mathrm{e} 7$ & 3.2 & $3.18 \mathrm{e} 8$ & 0.7 & 1.89 & $3.0 \mathrm{e} 8$ & 0.6 & 1 & 3.09 & 2.28 \\
\hline 4.3 & $3.67 \mathrm{e} 8$ & 3.2 & $6.20 \mathrm{e} 7$ & 6.4 & $2.63 \mathrm{e} 7$ & 3.2 & $3.88 \mathrm{e} 8$ & 0.6 & 1.91 & $3.0 \mathrm{e} 8$ & 0.6 & 1 & 3.06 & 2.31 \\
\hline 4.4 & $3.41 \mathrm{e} 8$ & 3.2 & $5.74 \mathrm{e} 7$ & 6.4 & $2.44 \mathrm{e} 7$ & 3.2 & $4.26 \mathrm{e} 8$ & 0.5 & 1.99 & $3.0 \mathrm{e} 8$ & 0.6 & 1 & 2.95 & 2.40 \\
\hline $4 \mathrm{a}$ & $4.22 \mathrm{e} 8$ & 3.0 & $7.37 \mathrm{e} 7$ & 6.0 & $3.02 \mathrm{e} 7$ & 3.0 & $7.56 \mathrm{e} 8$ & 0.8 & 1.0 & $3.0 \mathrm{e} 8$ & 0.6 & 1 & 2.73 & 2.95 \\
\hline $4 \mathrm{~b}$ & $4.33 \mathrm{e} 8$ & 3.4 & $7.09 \mathrm{e} 7$ & 6.8 & $3.09 \mathrm{e} 7$ & 3.4 & $5.21 \mathrm{e} 7$ & 0.8 & 5.24 & $3.0 \mathrm{e} 8$ & 0.6 & 1 & 3.50 & 2.34 \\
\hline $4 c$ & $4.25 \mathrm{e} 8$ & 3.2 & $7.17 \mathrm{e} 7$ & 6.4 & $3.04 \mathrm{e} 7$ & 3.2 & $1.29 \mathrm{e} 9$ & 0.8 & 1.0 & $4.15 \mathrm{e} 8$ & 0.6 & 1 & 2.90 & 2.36 \\
\hline $4 d$ & $4.12 \mathrm{e} 8$ & 3.2 & $6.95 \mathrm{e} 7$ & 6.4 & $2.95 \mathrm{e} 7$ & 3.2 & $1.10 \mathrm{e} 8$ & 0.8 & 5.24 & $6.51 \mathrm{e} 8$ & 0.6 & 1 & 2.96 & 2.21 \\
\hline
\end{tabular}

Bovy J., 2015, ApJS, 216, 29

Bovy J., Rix H.-W., 2013, ApJ, 779, 115

Bovy J., Rix H.-W., Schlafly E. F., Nidever D. L., Holtzman J. A., Shetrone M., Beers T. C., 2016, ApJ, 823, 30

Carlin J. L., et al., 2013, ApJ, 777, L5

Carlin J. L., et al., 2015, AJ, 150, 4

Cui X.-Q., et al., 2012, RAA, 12, 1197

De Silva G. M., et al., 2015, MNRAS, 449, 2604

Dehnen W., Binney J., 1998, MNRAS, 294, 429

Deng L.-C., et al., 2012, RAA, 12, 735

Freeman K., et al., 2013, MNRAS, 428, 3660

Gaia Collaboration et al., 2016, A\&A, 595, A2

Gilmore G., et al., 2012, The Messenger, 147, 25

Ho A. Y. Q., et al., 2016, preprint (arXiv:1602.00303)

Huang Y., et al., 2016, MNRAS, 463, 2623

Jeans J. H., 1915, MNRAS, 76, 70

Jeans J. H., 1919, Problems of cosmogony and stellar dynamics

Kaasalainen M., Binney J., 1994, MNRAS, 268, 1033

Kafle P. R., Sharma S., Lewis G. F., Bland-Hawthorn J., 2014, ApJ, 794, 59

Kunder A., et al., 2012, AJ, 143, 57
Lewis J. R., Freeman K. C., 1989, AJ, 97, 139

Liu C., et al., 2014, ApJ, 790, 110

Liu C., et al., 2017a, preprint, (arXiv:1701.07831)

Liu C., et al., 2017b, ApJ, 835, L18

Long R. J., Mao S., Shen J., Wang Y., 2013, MNRAS, 428, 3478

Majewski S. R., et al., 2013, ApJ, 777, L13

Martig M., Minchev I., Ness M., Fouesneau M., Rix H.-W., 2016, preprint (arXiv: 1609.01168)

McGill C., Binney J., 1990, MNRAS, 244, 634

McMillan P. J., 2011, MNRAS, 414, 2446

McMillan P. J., Binney J. J., 2008, MNRAS, 390, 429

Nordström B., et al., 2004, A\&A, 418, 989

Piff T., et al., 2014, MNRAS, 445, 3133

Portail M., Wegg C., Gerhard O., Martinez-Valpuesta I., 2015, MNRAS, 448, 713

Rich R. M., Reitzel D. B., Howard C. D., Zhao H., 2007, ApJ, 658, L29

Sanders J. L., Binney J., 2015, MNRAS, 447, 2479

Sanders J. L., Binney J., 2016, MNRAS, 457, 2107

Schönrich R., Aumer M., 2017, preprint, (arXiv:1704.01333)

Schönrich R., Binney J., Dehnen W., 2010, MNRAS, 403, 1829 
Schwarzschild M., 1979, ApJ, 232, 236

Steinmetz M., et al., 2006, AJ, 132, 1645

Syer D., Tremaine S., 1996, MNRAS, 282, 223

Tian H.-J., et al., 2015, ApJ, 809, 145

Tian H.-J., et al., 2016, preprint, (arXiv:1603.06262)

Wang Y., Zhao H., Mao S., Rich R. M., 2012, MNRAS, 427, 1429

Wang Y., Mao S., Long R. J., Shen J., 2013, MNRAS, 435, 3437

Widrow L. M., Gardner S., Yanny B., Dodelson S., Chen H.-Y., 2012, ApJ, 750, L41

Williams M. E. K., et al., 2013, MNRAS, 436, 101

Xu Y., Newberg H. J., Carlin J. L., Liu C., Deng L., Li J., Schönrich R., Yanny B., 2015, ApJ, 801, 105

Xue X. X., et al., 2008, ApJ, 684, 1143

Xue X.-X., Rix H.-W., Ma Z., Morrison H., Bovy J., Sesar B., Janesh W., 2015, ApJ, 809, 144

Yao S., et al., 2012, RAA, 12, 772

York D. G., et al., 2000, AJ, 120, 1579

Zhao G., Zhao Y.-H., Chu Y.-Q., Jing Y.-P., Deng L.-C., 2012, RAA, 12, 723

van der Kruit P. C., Searle L., 1981, A\&A, 95, 105

\section{APPENDIX A: THE UNCERTAINTY OF DISTANCE}

The distance uncertainty in Carlin et al. (2015) is propagated from the uncertainties in the effective temperature, surface gravity, and metallicity, which are provided by the LAMOST pipeline. However, we find that these uncertainties of the stellar parameters in the LAMOST catalogue are significantly overestimated (also see Schönrich \& Aumer (2017)). To obtain a more realistic assessment of distance uncertainty, we compare the distance for the $\mathrm{K}$ giant stars from Carlin et al. with that from Astraatmadja \& Bailer-Jones (2016) (hereafter ABJ16), who gave the Bayesian derived distance from the TGAS parallax (Gaia Collaboration et al. 2016). We obtain about 2000 common $\mathrm{K}$ giant stars with ABJ16 distance smaller than $1 \mathrm{kpc}$ and with relative uncertainty of ABJ16 distance smaller than 20\%. Figure A1 shows the distribution of the relative deviation of the two distances, $\left(D_{\mathrm{LAmosT}}-D_{\mathrm{ABJ} 16}\right) / D_{\mathrm{ABJ} 16}$. It can be seen that the Carlin et al. derived distance ( $\left.D_{\text {LAMOST }}\right)$ is systematically smaller by $10 \%$. Meanwhile, the dispersion of the relative deviation, measured with M.A.D. (median absolute deviation), is $22 \%$, which is a factor of 2 smaller than that claimed by Carlin et al. (2015).

This paper has been typeset from a $\mathrm{T}_{\mathrm{E}} \mathrm{X} / \mathrm{LAT}_{\mathrm{E}} \mathrm{X}$ file prepared by the author.

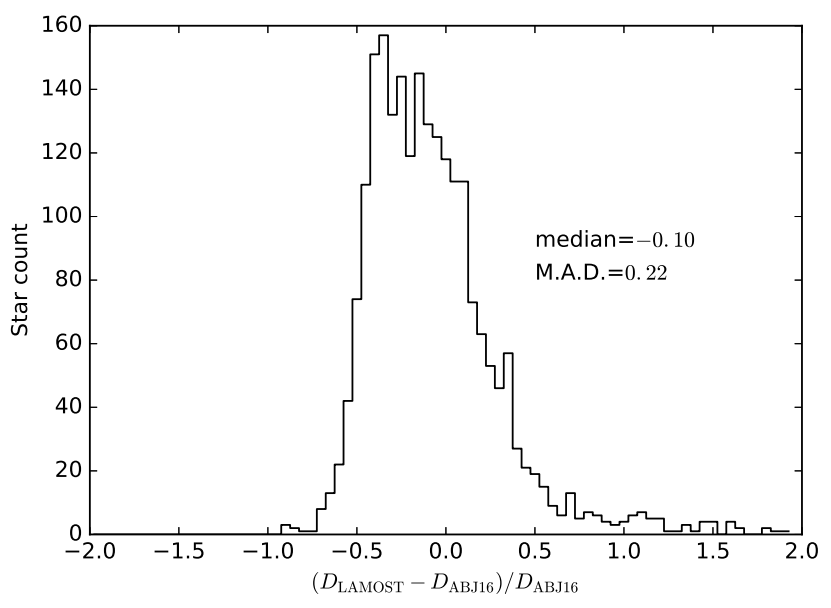

Figure A1. The black line shows the relative deviation between the distance $D_{\text {LAmosT }}$, which is derived by Carlin et al. (2015) and $D_{\mathrm{ABJ} 16}$, which is from ABJ16. The y-axis is the count of stars. 